\title{
The Role of Axonal Transport in Neurodegenerative Disease Spread: A Meta-Analysis of Experimental and Clinical Poliomyelitis Compares with Amyotrophic Lateral Sclerosis
}

\author{
Benjamin Rix Brooks
}

\begin{abstract}
ALS symptom spread results from local spread of the neuronal degeneration because contiguous areas are more quickly involved than non-contiguous areas. Local spread to contiguous areas of motor neuron dysfunction is faster at the brainstem, cervical and lumbar regions than spread to non-continguous areas. The time for caudal-rostral symptomatic spread of ALS to involve a distant region is a function of the distance of that region from the site of onset. The time for spread to the bulbar region is shorter following arm onset than leg onset. Spread to non-contiguous areas is faster within the spinal cord than from the spinal cord to the bulbar region. These kinetics are consistent with axonal transport of the etiological agent in a manner similar to spread of poliovirus in poliomyelitis patients. Spread from the bulbar region to the spinal cord, on the other hand, occurs faster than symptom spread from the limb region to the bulbar region in limb onset patients. This rapid limb involvement following bulbar onset is more dramatic in males compared with females. Females with leg onset, on the other hand, show more rapid involvement of the opposite leg, either arm or bulbar structures than males. Gender effects may determine the course of ALS depending on the original site of onset.
\end{abstract}

\begin{abstract}
RÉSUMÉ: La poliomyélite expérimentale et clinique comparée à la sclérose latérale amyotrophique : une métaanalyse. L'extension des symptômes dans la SLA résulte de la propagation locale de la dégénérescence neuronale, car les régions contiguës sont plus rapidement impliquées que les régions non-contiguës. La propagation locale de la dysfonction du neurone moteur à des régions contiguès est plus rapide dans le tronc cérébral, et dans la région cervicale et lombaire, que la propagation à des régions non-contiguës. Le temps de propagation des symptômes de SLA en direction caudale-rostrale pour atteindre une région éloignée est fonction de la distance de cette région du site initial. Le temps de propagation à la région bulbaire est plus court lorsque le site initial est le bras que lorsque c'est la jambe. La propagation à des régions non-contiguës est plus rapide dans la moelle épinière que de la moelle épinière à la région bulbaire. Cette cinétique est compatible avec le transport axonal de l'agent étiologique d'une manière similaire à la propagation du virus de la polio chez les patients atteints de poliomyélite. D'autre part, la propagation à partir de la région bulbaire à la moelle épinière survient plus rapidement que la propagation des symptômes à partir de la région des membres à la région bulbaire chez les patients avec atteinte initiale aux membres. Cette atteinte rapide des membres suite à une atteinte bulbaire initiale est plus dramatique chez les hommes que chez les femmes. Les femmes avec atteinte initiale à la jambe manifestent une atteinte plus rapide de la jambe opposée, de l'un ou l'autre bras ou des structures bulbaires que les hommes. Des effets dus au sexe peuvent déterminer l'évolution de la SLA selon le site initial de l'atteinte.
\end{abstract}

Axonal transport may be the means for disease spread of neurodegenerative diseases such as amyotrophic lateral sclerosis. A rapidly progressive motor neuron disease in which axonal transport of the causative agent is important in the spread of dis- ease within the central nervous system is poliomyelitis caused by the picornavirus, poliovirus. Hematogenous spread of poliovirus to the central nervous system allows for the multifocal onset; however, there is evidence that once poliovirus is innoculated

From the Amyotrophic Lateral Sclerosis Clinical Research Center, Neurology Department, University of Wisconsin-Madison Medical School; the Neurology Service,William S Middleton Memorial Veterans Administration Medical Center, and the MDA Midwest Regional ALS Treatment and Research Program, Madison, Wisconsin

Reprint requests to: Benjamin Rix Brooks, MD, Chief, Neurology Service, Wm S Middleton Memorial VA Hospital, 2500 Overlook Terrace B-6112, Madison, Wisconsin, U.S.A. 53705 
naturally by hematogenous spread or experimentally by direct injection into the central nervous system, spread of poliovirus and development of symptoms is due to axonal transport. ${ }^{1-3}$ In poliomyelitis, symptomatic weakness by the MRC scale occurs after loss of $70-80 \%$ of neurons. 4.5

We analyzed the development of paralysis in poliomyelitis patients following onset of fever and pain. Cumulative paralysis regardless of site of onset in four epidemics separated by 50 years and many miles showed remarkable similarities. We pooled the data from 78 patients with leg onset of poliomyelitis in a meta-analysis and determined by a modification of life table analysis that the spread to the opposite leg was significantly faster than spread to either arm or to involve the bulbar region. In the analysis of data from a large group of patients with leg onset of poliomyelitis, we could see that spread of clinical symptoms in a population of patients showed a gradient in time and proportion of patients involved from the site of initial involvement.

We proceeded to employ this method to analyze the natural history of symptom spread in large groups of patients with amyotrophic lateral sclerosis of the Charcot variant and found different rates of symptom spread depending on the site of onset and the gender of the patient ${ }^{6}$ The time of the first symptom may not define the true start of the disease process. However, each succeeding symptom due to lower motor neuron loss will accrue according to a function dependent upon the time, relative to the onset of the first symptom, that it takes for motor neuron groups to lose neurons down to the $20 \%$ threshold at which symptoms are obvious to the patient.

\section{Topographical Effects on Symptom Development in ALS}

Patients who developed ALS were followed for up to seven years. Complete information on the accrural of bulbar and limb symptoms was available on 702 patients who had completed at least two follow-up questionnaires.

Limb Onset Symptom accrural in the opposite leg of 174 unilateral leg onset ALS patients was significantly faster than symptom accumulation in both the contralateral $[\mathrm{p}<0.03]$ and ipsilateral [p $<0.03$ ] arm [Figure 1A]. The symptom accrural in opposite arm compared with contralateral leg or ipsilateral leg respectively of 213 unilateral arm onset ALS patients was significantly $[\mathrm{p}<0.001]$ faster than bulbar symptom accrural during the first five years of disease [Figure 1B]. Moreover, compared to the accrural of bulbar symptoms, clinical weakness in other limbs [opposite leg, ipsilateral arm, and contralateral arm] of ALS patients following unilateral leg onset was significantly faster [p < 0.04] for the first five years of disease.

Bulbar Onset Cranial motor neuron symptom accrural in unilateral arm onset ALS patients was significantly [ $p<0.04$ ] faster than bulbar symptom accrural in unilateral leg onset ALS patients at 2,3, and 4 years of disease [Figure 1C]. In 96 bulbar ALS patients with speech onset, swallowing symptom accrural was significantly $[p<0.001]$ faster than both arm and leg symptom accrural for the first four years of disease [Figure 1D]. Presenting the course of ALS in this fashion we can see for the first time that segmental symptom development in an ALS population [arm to contralateral arm, leg to contralateral leg, speech to swallowing] occurred faster and to a greater extent than distal symptom accrural [arm to ipsi- or contra-lateral leg, leg to ipsior contra-lateral arm, speech to arm or leg]. Moreover, symptom accrural within the spinal cord occurred faster than bulbar symptom accrural in ALS patients with either unilateral arm or leg onset. However, limb symptom development occurred faster subsequent to bulbar onset than bulbar symptom development following limb onset.

\section{Gender Effects on Symptom Development in ALS}

We determined the effects of gender on the spread of symptoms in patients with ALS as a function of the site of onset of the disease. Compared with male ALS patients, female ALS patients early after leg onset developed significantly [p $<0.02]$ increased opposite leg symptoms, ipsilateral arm symptoms, contralateral arm symptoms and bulbar symptoms. [Figure 1E and $[F]$. A marked contrast occurred in male ALS patients early following bulbar onset in that they manifested significantly $[\mathrm{p}<$ $0.01]$ more symptom spread to arms than was seen in females. The course of symptom development in ALS patients with arm onset was independent of the gender of the patient.

\section{Discussion}

These findings suggest that ALS symptom spread results from local spread of the neuronal degeneration because contiguous areas are more quickly involved than non-contiguous areas. The local spread to contiguous areas of motor neuron dysfunction is faster at the brainstem, cervical and lumbar regions. Spread to non-contiguous areas is faster within the spinal cord than from the spinal cord to the bulbar region. Nevertheless, the time for spread to the bulbar region is shorter following arm onset than leg onset. Thus, the time for spread to involve a distant region is a function of the distance of that region from the site of onset. Spread from the bulbar region to the spinal cord, on the other hand, occurs in a larger percentage of patients at 12 , 24 , and 36 months following bulbar onset than spread from the limb region to the bulbar region in limb onset patients. The more rapid limb involvement following bulbar onset is more dramatic in males compared with females. However, the more rapid onset of opposite leg, either arm or bulbar involvement in females compared with males after leg onset indicates that gender effects may determine the course of ALS depending on the original site of onset.

These observations do not immediately allow distinction among degenerative, toxic or infectious etiologies. Spread of symptoms may reflect susceptibility to a particular etiology, not simply the mode of spread of that etiology. For instance, overexercised or injected limbs may determine the initial localization of hematogenously delivered poliovirus to particular spinal segments. ${ }^{7,8}$ Blood-borne toxins, such as botulinum toxin, may

Figure I - Cumulative Percent Symptomatic ALS Patients following Limb or Bulbar Onset.

The development of the proportion of patients who display opposite limb or ipsilateral and contralateral distant limb involvement or bulbar involvement following disease onset is computed for patients with leg $[A]$, arm [B], bulbar [C] or speech [D] onset. ${ }^{6}$ The effect of gender on arm [E] or bulbar [F] symptom development is studied separately in females and males after leg onset. Asterisks denote statistically significant [ $p<0.05$; life table analysis] differences between limb and bulbar involvement $[A, B]$ or between arm and leg involvement $[C]$ or between arm and leg involvement $[C]$ or between speech onset and arm or leg onset [D] or either leg onset and contralateral arm [E] or bulbar [F] involvement. 

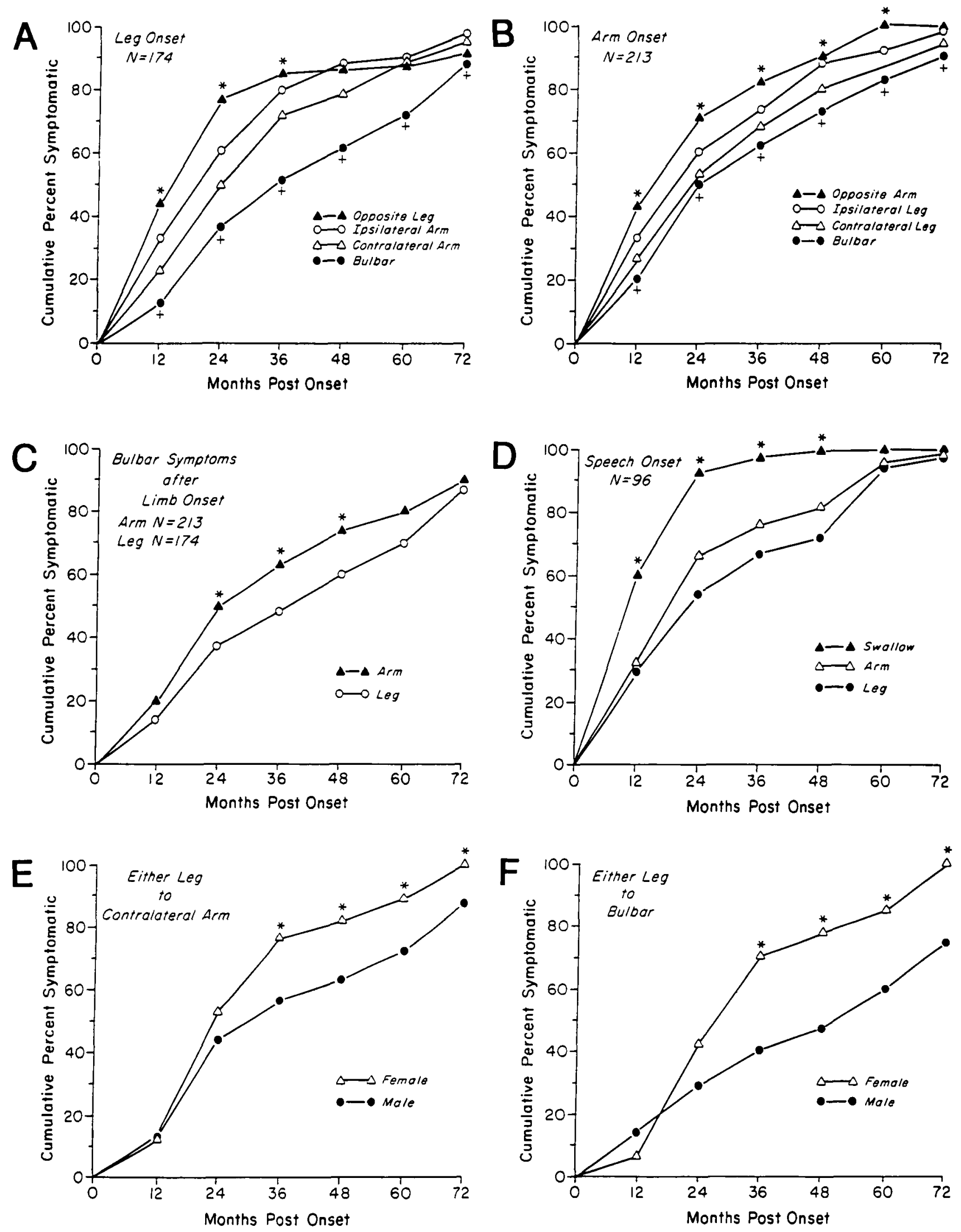

Figure I 
affect susceptible ocular motor neurons in the brainstem before the phrenic motor neurons. However, the spread of ALS symptoms beginning in the spinal cord appears to be statistically a function of the distance of the site of origin from the secondary and tertiary sites of involvement similar to what we have observed in poliomyelitis. By analogy therefore, there is a strong possibility that spread of ALS symptoms originating at the spinal level involves axonal transport. The more rapid rostal-caudal involvement in ALS after bulbar onset compared with the caudal-rostral involvement in ALS after spinal involvement might argue for a different mechanism. Onset of symmetrical spinal cord anterior horn disease mediated by means of rostal-caudal spread of infectious agents via the central canal has been previously demonstrated in experimental animals. ${ }^{9}$ Statistically, symmetrical arm involvement occurs in males faster than females following bulbar onset. This type of spread in ALS may be either due to axonal transport or to a mechanism involving the central canal. When spread is displaying a gender effect such as we observe, then the gender effect may result from different mechanisms or from a gender effect on a common mechanism.

This study of the spread of ALS symptoms has provided a probability statement for symptom spread as a function of site of origin and gender. The precise mechanisms of symptom spread in ALS are not understood at present. Nevertheless, more careful study of large groups of ALS patients, that have a particular site of onset, by quantitative clinical and electrophysiological techniques will provide the timeline for motor unit involvement. The pathological study of patients with different sites of onset may provide information on the anatomical substrates for such spread. It is crucial to carefully and quantitatively analyze at autopsy different regions of the nervous system in ALS patients who die at different times following onset of disease at different sites. This analysis of symptom spread constitutes only the first step in a quantitative analysis of the pathogenesis of ALS.

\section{ACKNOWLEDGEMENTS}

The clinical studies reported in this report were made possible by Muscular Dystrophy Association grants to support studies in the Experimental Treatment of ALS and the MDA Midwest Regional ALS Research and Treatment Program [BRB]. Additional partial support was provided by the Department of Veterans Affairs Merit Review Grant Program [BRB].

\section{REFERENCES}

1. Bodian D. A reconsideration of the pathogenesis of poliomyelitis. Am J Hyg 1952; 55: 414-438.

2. Russell WR. Paralytic poliomyelitis: The early symptoms and the effect of physical activity on the course of the disease. $\mathrm{Br}$ Med J $1949 ; 1: 465-471$.

3. Jubelt B, Narayan O, Johnson RT. Pathogenesis of human poliovirus infection in mice. II. Age-dependency of paralysis. J Neuropathol Exp Neurol 1980; 39: 149-159.

4. Sharrard WJW. The distribution of permanent paralysis in the lower limb in poliomyelitis. A clinical and pathological study. J Bone Joint Surg 1955; 37B: 540-558.

5. Sharrard WJW. Poliomyelitis: The distribution of the Paralysis. In: Garling ER, Ross JP, eds. British Surgical Practice: Surgical Progress, London, Butterworth 1956; 83-103.

6. Brooks BR, DePaul R, Tan Y-D, Sanjak M, Sufit RL, Robbins R Chapter 18. Motor Neuron Disease. In: Schoenberg BS, Porter $\mathrm{R}$, eds. Controlled Clinical Trials in Neurology, Norwood, Marcel Dekker 1990: 249-281.

7. McAlpine D, Buxton PH, Kremer M, Cowan DJ. Acute poliomyelitis with special reference to early symptomatology and contact histories. Br Med J 1947; 2: 1019-1023.

8. Howe HA, Bodian D. Neural Mechanisms in Poliomyelitis, New York, Commonwealth Fund 1942; 1-234.

9. Jackson AC, Moench TR, Griffin DE, et al. The pathogenesis of spinal cord involvement in the encephalomyelitis of mice caused by neuroadapted Sindbis virus infection. Lab Invest 1987; 56 : 418-423. 